\title{
Schulungs-CD TarMed
}

Inhalt:

- Ausgangslage

- Entstehungsgeschichte

- Tarifaufbau (Tarifberechnungen)

- Fallbeispiele/Abrechnungsbeispiele

- Tarifbrowser

FMH-Mitglieder können die CD zum Preis von Fr. 40.- (zuzüglich Fr. 5.50 Porto und Verpackung) beim FMH Tarifdienst bestellen. Aus administrativen Gründen können nur Bestellungen per Fax entgegengenommen werden. Lieferfrist etwa 14 Tage. Die Auslieferung erfolgt per Nachnahme. Für Nicht-Mitglieder beträgt der Preis Fr. 60.- (exkl. Porto und Verpackung).

Hinweis: Die Fallbeispiele basieren noch auf der Version TARMED 2.2. Die Fallbeispiele in der Grundversorgung werden aber durch die Änderung zu TarMed 3 nicht tangiert.

Systemvoraussetzung: Intel Pentium oder vergleichbarer Prozessor, mind. 8 MB RAM, Microsoft Windows 95/98/NT 4.0, Grafikkarte mit einer Auflösung von mindestens $800 \times 600$ und 256 Farben (empfohlen: High-

Color oder TrueColor), CD-ROM Laufwerk; Internet Explorer 4.0.

\section{Bestelltalon}

Name:

Vorname:

Adresse:

Unterschrift:

Anzahl CDs:

FMH Tarifdienst, Fax 0419210586

\section{Hilfskasse für Schweizer Ärzte}

\section{Eingegangene Spenden}

Vom 1. April bis 30. Juni 2000 sind 146 Spenden im Gesamtbetrag von Fr. 7488.- eingegangen. Der Stiftungsrat der Hilfskasse für Schweizer Ärzte freut sich sehr, diese Gaben bekanntgeben zu dürfen, und dankt allen Spenderinnen und Spendern recht herzlich. Damit die Spenden in voller Höhe den Destinatären zukommen, haben wir uns entschlossen, für Spenden unter Fr. 500.- auf den Versand von persönlichen Dankschreiben zu verzichten. Wir hoffen sehr, dass diese Massnahme bei Ihnen auf Verständnis stösst.

Der Kassier des Stiftungsrates: Dr. U. Straumann 


\section{CD de formation TarMed}

Contenu:

- Situation initiale

- Historique

- Conception du tarif (calculations)

- Exemples de cas/exemples de facturation

- Navigateur tarifaire

Les membres de la FMH peuvent commander ce CD-ROM auprès du Service tarifaire de la FMH pour la somme de Fr. 40.- (plus Fr. 5.50 de frais de port et d'emballage). Pour des raisons administratives, seules les commandes par fax peuvent être prises en compte. Le délai de livraison est d'environ 15 jours. La livraison se fait contre remboursement. Pour les non-membres de la FMH, le prix du CD-ROM est de Fr. 60.- (sans les frais de port et d'emballage).

N.B.: les exemples de cas se basent encore sur la version 2.2 du TarMed. Ceux concernant la médecine de premier recours ne sont pas touchés par les modifications de la version 3 du TarMed.

Système informatique nécessaire: Intel Pentium ou processeur similaire, au moins 8 MB de RAM, Microsoft Windows 95/98 NT 4.0, carte graphique avec une résolution d'au moins 800×600 et 256 couleurs (conseil: davantage de couleurs ou couleur vraie), lecteur de CD-ROM; Internet Explorer 4.0.

se

\section{Bulletin de commande}

Nom:

Prénom:

Adresse:

Signature:

Nombre de CD-ROM désiré:

Service tarifaire FMH, fax 0419210586

\section{Caisse de secours des médecins suisses}

\section{Contributions reçues}

Du $1^{\text {er }}$ avril au 30 juin 2000, nous avons reçu 146 dons d'une valeur totale de Fr. 7488.-. Le Conseil de fondation de la Caisse de secours des médecins suisses est très content de pouvoir publier ces chiffres et tient à remercier vivement tous les donateurs. Afin que les contributions puissent être versées entièrement aux destinataires, nous avons pris la décision de renoncer à l'envoi de lettres de remerciement pour les dons inférieurs à Fr. 500.-. Nous espérons que les donateurs feront preuve de compréhension et nous leur adressons encore une fois nos remerciements. 